\title{
„Für einen Aktivisten wie mich muß es in einem sozialistischen Staat doch effektive Medikamente geben"
}

\author{
Psychopharmaka und Konsumenteninteresse in der DDR
}

\section{Viola Balz}

\begin{abstract}
„In a Socialist State it Should be Possible for Activists like me to Receive Effective Medication." Psychotropic Drugs and Consumer Interests in the GDR

This article investigates how much influence consumers could have in the GDR during the 1960s by means of a case study of a patient striving towards an apparently reasonable psychiatric treatment through his actions in- and outside the clinic. The patient tried to obtain an effective therapy by referring to his model socialist work and life. The former patient also subsequently acted as a consumer via different activities, both within the GDR and abroad. Finally, against the background of different theories of patient and consumer history, this article will discuss how consumer historical approaches can be useful for studying a socialist state.
\end{abstract}

Keywords: Psychotropic drugs, consumer, patient, GDR, psychiatry

Schlüsse/wörter: Psychopharmaka, Konsument, Patient, DDR, Psychiatrie

Auf den Friedhof habe ich mich schon oft gewünscht weil es in unserem Arbeiterund Bauernstaat auch keine Mittel gibt um solche Krankheiten zu heilen. ${ }^{1}$

Diese Zeilen, die der Patient, den ich im Folgenden Hans Arnold nennen werde, im Jahr 1963 an seinen ehemals behandelnden Psychiater in der Berliner Charité schrieb, verdeutlichen eindrücklich den vom Patienten empfundenen Mangel an wirksamen Arzneimitteln in der DDR. Hans Arnold wollte sich jedoch nicht damit abfinden, dass sich seine psychische Gesundheit, wie ihm die Ärzte der Charité immer wieder versicherten, durch kein Medikament mehr verbessern ließe. Er war ein mehrfach prämierter Vorarbeiter und engagierter Sozialist und nahm die Versprechen der sozialistischen Gesundheitspolitik ernst: Schutz und Stärkung der Gesundheit durch Prävention, betriebsärztliche Versorgung und einen guten Sozialversicherungsschutz für die Arbeiter als Grundlage der sozialistischen Gesellschaftsordnung. Wie ich im Folgenden zeigen werde, 
wurde Arnolds Erwartung auf bestmögliche Behandlung jedoch durch die permanente Verschlechterung seines Zustands getrübt. So beschloss er schließlich, sich eigenständig um eine bessere Medikamentenversorgung zu bemühen. Die Berliner Universitätsklinik setzte er durch eine Vielzahl von Briefen, die sich sorgsam abgeheftet in seiner Krankenakte finden, über seine Korrespondenz mit verschiedenen Gesundheitseinrichtungen im In- und Ausland in Kenntnis. Dabei löste er mitunter heftige Reaktionen der Ärzte aus.

Die Geschichte von Herrn Arnold möchte ich unter einem patientengeschichtlichen Fokus betrachten und fragen, inwieweit Arnold dabei auch als Konsument agierte. ${ }^{2}$ Als Quelle dient mir die Krankenakte von Hans Arnold, die neben der Krankengeschichte zahlreiche Briefe von Arnold an die Klinik enthält. Zunächst werde ich mit einer Analyse seiner Krankengeschichte beginnen. An Arnolds Behandlung in der Charité lässt sich der Umgang mit dem Mangel an wirksamen Psychopharmaka in der DDR exemplarisch beschreiben. Ich werde diesen Abschnitt mit der Sichtweise der Ärzte auf den Patienten beenden. Anschließend werde ich den Blick von Hans Arnold auf sich selbst, soweit er in den Akten aufscheint, skizzieren.

Im Zentrum meines Interesses steht dabei die Frage, inwieweit Hans Arnold als Konsument agierte, das heißt als ein Patient, der die (bestmöglichen) Behandlungsoptionen aktiv auswählte und nach unabhängigen Informationen verlangte. Dabei möchte ich erstens darlegen, wie Arnold seinen Anspruch, als Sozialist der ersten Stunde eine besonders gute Therapie zu bekommen, selbstbewusst vorträgt. Diese Hinweise auf seine vorbildliche Arbeits- und Lebensweise führten vermutlich in der Charité zu einer Behandlung mit zum Teil nur sehr eingeschränkt verfügbaren Psychopharmaka. Zweitens möchte ich untersuchen, inwieweit Arnold nach seiner Klinikentlassung durch seine grenzüberschreitenden Aktivitäten im In- und Ausland seine Ansprüche auf adäquate Behandlung weiterverfolgte. Drittens möchte ich skizzieren, wie Arnold diese Konsumentenposition stabilisierte, indem er einzelne Parteikader hinzuzog, die ihn unterstützten. Viertens möchte ich abschließend reflektieren, welche Besonderheiten an diesem Beispiel eines aktiven, eigensinnigen Patienten im Sozialismus deutlich werden.

Um die Fallgeschichte besser einordnen zu können, werde ich zunächst auf den Stand der Patientengeschichte im Verhältnis zu konsumentengeschichtlichen Ansätzen eingehen. Anschließend werde ich aufzeigen, in welcher Weise sich die Krankenakte als Quelle für eine solche Geschichte anbietet.

\section{Patientengeschichte, Konsumenteninteresse und Krankenakten}

Der Ruf nach einer Geschichte des Patienten ist keineswegs neu (Porter 1985, Wolff 1998). Der geforderte Perspektivwechsel ist aber keineswegs einfach - 
denn für eine history from below mangelt es oft an Quellen. Auch Krankenakten stellen keine umfangreichen, vordiskursiven und/oder authentischen Überlieferungen bereit. Einen methodischen Zugang, auf den bereits Roy Porter aufmerksam gemacht hat, bietet die dialogische Form, in der institutionelle Patientengeschichten oft die Interaktion mit dem Arzt überliefern (Porter 1985: 175). Ob sich in den dialogisch stark geprägten Aufzeichnungen der Krankenakte nun, wie Porter annimmt, Spuren des Patientenerlebens finden oder die Äußerungen der Patienten erst von dem Zusammentreffen mit dem Arzt und damit der Institution produziert werden (Gillis 2006), ist inzwischen eine müßige Frage. Denn in jedem Fall haben in machtgeprägten Settings auch schwache historische Akteure wie Arbeiter, Bauern oder Patienten Spielräume für eigensinnige Handlungen (Foucault 1987). Dieser Eigensinn ist, wie insbesondere Alf Lüdtke betont, nicht per se als widerständig zu verstehen. Vielmehr müsse die historische Analyse darauf fokussieren, wie Menschen den Geschehnissen einen eigenen Sinn verliehen haben (Lüdtke 1993). Gerade für eine Geschichte der DDR ist die Nützlichkeit des Eigensinnkonzepts herausgearbeitet worden. Die totalitären Gestaltungsansprüche des Staates haben sich, wie beispielsweise Thomas Lindenberger zeigt, nicht umsetzen lassen, da die Bevölkerung die staatlichen Handlungsmaximen in ihrem eigenen Sinn interpretierte und teilweise auch dagegen aufbegehrte (Lindenberger 1999: 14).

Um die Spuren eines solchen Eigensinns zu rekonstruieren, bietet sich als Quelle die Krankenakte ganz besonders an. Nicht selten enthalten die Dossiers, wie im Fall von Hans Arnold, von Patienten selbst verfasste Briefe und Dokumente oder sie referieren die von den behandelnden Ärzten erfasste Rede des Patienten weitgehend. Die in diesen Quellen enthaltenen Patientenaussagen sind zwar durch den ärztlichen Blick gefiltert, in dem beispielsweise einige Passagen hervorgehoben wurden, andere wiederum dafür zu unwichtig erschienen, weshalb diese Dokumente keinesfalls als vordiskursive Aussagen von Patienten betrachtet werden dürfen. Die Krankenakte selbst bietet uns per definitionem nur einen Ausschnitt jener Erlebnisse der Patienten, die diese dem medizinischen Personal mitteilen wollten, die also in jedem Fall auf die Institution bezogen blieben. Auch wenn sich Patienten in der DDR durchaus einer sozialistischen Rhetorik in Briefen an die Klinik bedienten, sind diese Quellen von den Eingaben an das Ministerium für Gesundheitswesen zu unterscheiden. Mit diesen Schreiben versuchten die Bürger direkt den Bedarf an bestimmten Medikamenten anzuzeigen. ${ }^{3}$

Unabhängig von jeder Quellendiskussion sind im Bereich der Arzneimittelgeschichte patienten- und konsumgeschichtliche Ansätze kaum erforscht. Während in der Medizingeschichte zahlreiche Studien mit patientengeschichtlichem Fokus vorliegen, blieb eine patientengeschichtliche Rekonstruktion der Arzneimittelgeschichte bisher ein Desiderat (Balz 2010b: 156, Eckart/Jütte 2007: 185). ${ }^{4}$ Gleichzeitig wurden jedoch vereinzelt 
Forderungen laut, den Patientenstandpunkt um die Perspektive des Konsumenten zu ergänzen, das heißt die Nachfrage und Selbstmedikationen von Patienten in die historische Untersuchung einzubeziehen (Van der Geest/ Whyte/Hardon 1996: 166). Das Konzept des Konsumenten ist vor dem Hintergrund einer Patientengeschichte überaus reizvoll, da es den analytischen Fokus auf den aktiven, eigensinnigen Patienten richtet und sich zugleich für eine originäre Geschichte der Nachkriegsgesellschaft anbietet: Der Patient ist nicht länger der vom Arzt abhängige Partner in der Interaktion, sondern zeichnet sich dadurch aus, dass er aktiv medizinische Leistungen auswählt und sich diese aneignet. Hierfür verlangt er nach umfassender, möglichst unabhängiger Information (Donohue 2006). Diese Eigenaktivität von Bürgern wurde insbesondere in einigen Untersuchungen zur Regulation von Arzneimitteln herausgearbeitet. ${ }^{5}$ Die bislang vorliegenden Studien untersuchen die Rolle des Konsumenten jedoch nur für liberale kapitalistische Staaten. Studien zur Konsumgeschichte der DDR blieben hingegen, so Ina Merkel, lange Zeit eine Rarität (Merkel 1999: 9). ${ }^{6}$ Dies war nicht zuletzt den Schwierigkeiten geschuldet, innerhalb einer sozialistischen Gesellschaftsordnung von Konsum sprechen zu können, da diese als „Mangelgesellschaft“ galt (Merkel 1999: 24). Dies verkennt jedoch, dass es in der DDR durchaus eine Konsumkultur und politik gab. Anders als andere osteuropäische Staaten galt die DDR schon seit den 1960er Jahren als Massenkonsumgesellschaft (Kaelble 1997: 192). ${ }^{7}$ Wie Stephan Merl ausführt, stieg seit 1957 der Konsum nach längerer Stagnation in der Nachkriegszeit stetig an. Auf dem 5. Parteitag der SED 1958 versprach Ulbricht der Bevölkerung, die BRD hinsichtlich der Versorgung mit Konsumgütern bis 1961 einzu- beziehungsweise sogar zu überholen. Der Wettstreit mit der BRD, so folgert Merl, wurde also von der Ebene des politischen Systems auf die Ebene des Konsums verlagert (Merl 1997: 211). Anders als in Westdeutschland war dieser Konsum aber vor allem von der Politik gesteuert und von der Entwicklung der Wirtschaft abgekoppelt (Merl 1997: 207). Die Konsumgüterindustrie erfolgte dabei planwirtschaftlich und brauchte an keiner Stelle Rücksicht auf die Wünsche der Verbrauer zu nehmen (Merl 1997: 232). Die mangelnde Vielfalt der Produktpalette sollte unter anderem dadurch ausgeglichen werden, dass die staatlichen Planungskommissionen versuchten, auf den Geschmack der Bevölkerung einzuwirken. Wie Merkel betont, ist es wichtig, an kapitalistischen Staatsformationen orientierte Vorstellung von einer freien Wahl eines souveränen Konsumenten aus einem Überangebot an Waren als zentrales Merkmal von Konsumgeschichte zu überdenken. Der Anspruch der DDR-Staatsführung war auch nicht der einer Konsumgesellschaft, sondern der einer Kulturgesellschaft. Gleichwohl artikulierte die Bevölkerung durchaus abweichende Konsumbedürfnisse. Zielführender ist hier nach Merkel der Begriff der Konsumkultur, der das widersprüchliche Verhältnis von Konsumpolitik und Konsumverhalten der Bevölkerung kennzeichnete (Merkel 1999: 26-27). 
Die beschriebene Beschränkung der Konsumgüter in der DDR lässt sich auch auf den Bereich der Arzneimittel übertragen. Wenngleich die Möglichkeit eines vermeintlich freien Medikamentenkonsums selbst in den westlichen Ländern weitgehend Fiktion blieb, konnten die Bürger hier dennoch aus einem großen Medikamentenangebot wählen. Eine Konsumentengeschichte, die auf die besondere Situation in der DDR eingeht, muss jedoch berücksichtigen, dass dem Konsum von Arzneimitteln im Sozialismus enge Grenzen gesetzt wurden, da sich der Markt nicht durch Angebot und Nachfrage, sondern durch staatliche Vorgaben regulierte und zugleich die Arzneimittelversorgung auf die als unbedingt notwendig erachteten Produkte beschränkt wurde. ${ }^{8}$ Wie ich zeigen werde, eröffnete sich Hans Arnold aber auch innerhalb der DDR ausreichend Spielräume, um sich aktiv um die Verfügbarkeit neuer Arzneimittel $\mathrm{zu}$ bemühen. Bereits Roy Porter hat herausgearbeitet, dass sich individuelles Gesundheitsverhalten nicht auf medizinische Behandlung beschränkt. Es schließt Vorkehrungen mit ein, die unter anderem Vorsorge, Deutungsmodelle und soziale Vernetzung umfassen (Porter 1985: 193). Wie Konzepte der Konsumentengeschichte darüber hinaus betonen, beschränkt sich Konsum nicht auf den Verbrauch von Gütern. Vielmehr gestalten die Akteure mit dem Konsum bestimmter Güter aktiv ihren Lebensstil (Applbaum 2006). Solchen theoretischen Modellierungen zufolge müssen auch medizinische Angebote wie beispielsweise der Psychopharmakakonsum auf ihre Bedeutungs- und Sinnzuschreibungen befragt und untersucht werden, die mit einer (auch imaginären) Teilhabe an einem gewissen Lebensstil und einer Gemeinschaft verbunden ist (Henderson/ Petersen 2003: 3).

In der folgenden Schilderung von Hans Arnolds Geschichte möchte ich die Bedeutung seines Medikamentenkonsums für ihn selbst skizzieren und dabei der Frage nachgehen, inwiefern Arnold dabei auch als Konsument agierte. Ich werde herausarbeiten, welche Sinn- und Bedeutungszuschreibungen Arnold mit dem Psychopharmakakonsum verband und an welcher Gemeinschaft er mit Hilfe dieses Konsums teilhaben wollte. Zunächst möchte ich jedoch mit Arnolds Krankengeschichte beginnen, an der sich gängige Behandlungsmöglichkeiten mit Psychopharmaka in dieser Zeit paradigmatisch aufzeigen lassen.

\section{Eine Vorzugsbehandlung? Hans Arnold als Patient der Charité}

$\mathrm{Zu}$ Beginn seiner Patientengeschichte ist Hans Arnold 59 Jahre alt. Eingewiesen von der Nervenpoliklinik wurde er am 8. Juni 1960 auf der psychiatrischen Abteilung der Psychiatrischen und Nervenklinik der Ostberliner Charité aufgenommen. Seine Lebensgeschichte fassten die Ärzten in der 
Exploration wie folgt zusammen: Bis zum Alter von 14 Jahren sei er zur Grundschule gegangen und anschließend zunächst in der Landwirtschaft, dann als Heizer angestellt worden. In den 1920er Jahren sei er häufig arbeitslos oder nur teilweise beschäftigt gewesen. Nach dem Krieg habe er als Gießkranführer gearbeitet, eine Tätigkeit, die er nach eigenen Angaben ausgesprochen gerne ausführte. 1953 wurde er dann zunehmend gehemmt und sei in den Arbeitspausen nur noch stumm in den Wald gegangen. Wie seine Ehefrau in der Befragung angab, blieb er in den Pausen schließlich ganz auf dem Kran. Seine Arbeitskollegen, denen dieser Zustand auffiel, veranlassten schließlich, dass Arnold in eine psychiatrische Landesklinik eingewiesen wurde, wo man ihn zunächst sechs Wochen lang mit Elektroschocks behandelte. Nach seiner Entlassung aus der Anstalt habe Arnold seine Arbeit ohne größere Unterbrechungen bis 1957 wieder aufgenommen. In diesem Jahr führte eine Verschlechterung seines Befindens erneut zu einer Einweisung, aus der er als „ungebessert" entlassen wurde. Anschließend gab er seinen Beruf als Kranführer auf. Im Oktober 1959 führten seine zunehmende Gehemmtheit, Schlaflosigkeit und traurige Verstimmung $\mathrm{zu}$ fast ununterbrochenen Klinikaufenthalten. ${ }^{9}$

Über die Diagnose waren sich die Ärzte zunächst keineswegs sicher. So beschrieben sie zwar, dass der Patient sich gehemmt und verlangsamt verhalte. Sie führten den momentanen Zustand aber vor allem auf den stetigen Arzneimittelgenuss von täglich ein bis zwei Schlaftabletten in den vorausgegangenen drei Jahren zurück - ein Konsum, den der behandelnde Arzt als missbräuchliche Verwendung beschrieb. Arnolds vorläufige Diagnose lautete folglich „Organischer Hirnschaden nach Schlafmittelabusus mit depressiv gefärbten psychischen Veränderungen“. Eine Woche nach seiner Einlieferung wurde Arnold schließlich dem Chefarzt der Klinik, Karl Leonhard, vorgestellt. Leonhard galt und gilt heute noch als berühmter Psychopathologe. Er hatte ein eigenes, weltweit bekanntes System zur Klassifikation von Psychosen entwickelt (Balz/Hoheisel 2011). Leonhard gegenüber wiederholte Arnold, dass er gehemmt, still und schlaflos sei, bejahte aber, dass er Interesse und Entschlusskraft habe. Leonhard stellte schließlich die Diagnose der „periodisch reinen Melancholie“. Die wichtigsten Symptome der Melancholie, so Leonhard in seinem Buch „Einteilung der endogenen Psychosen“, seien gedrückte Stimmung, teilnahmsarmes Verhalten und eine psychomotorische Hemmung, die zwar depressiv wirken könne, aber nicht die Traurigkeit einer echten Depression aufweise. Über die Therapiemöglichkeiten wird in Leonhards Monographie jedoch nichts weiter berichtet (Leonhard 2003). So gibt das Werk auch keinen Hinweis darauf, warum die Ärzte kurz nach Leonhards Diagnose mit einer Imipraminverordnung begannen. Bei Lektüre der Exploration wird jedoch deutlich, dass die Ärzte von einer Behandlung des Patienten mit dem Medikament in einer anderen Klinik berichteten, deren Erfolge sie jedoch nicht näher spezifizierten. 
Imipramin (Handelsname in Westdeutschland: Tofranil) war das erste Antidepressivum überhaupt. 1958 wurde es von Roland Kuhn in der Schweiz erprobt und als antidepressiv wirksam beschrieben (Kuhn 1958). Für den ostdeutschen Gebrauch ist jedoch zu berücksichtigen, dass die Nachentwicklung durch die ungarischen Vereinigten Heil-und Nährmittelwerke Zeit in Anspruch nahm. 1962 stand das Generikum Melipramin als Import dem sozialistischen Bruderstaat zur Verfügung (Staatliches Institut/Gerecke 1962: 171), nachdem die begehrte Neuentwicklung bereits seit 1959 aus dem kapitalistischen Westen importiert und auch in der Ärzteschaft diskutiert wurde (Aresin 1960). Leonhard selbst hatte das Manuskript des Behandlungsberichts der Leipziger Klinik für die Zeitschrift Psychiatrie, Neurologie und medizinische Psychologie gegengelesen. Ihm war das neue Medikament somit spätestens Mitte 1960 bekannt. ${ }^{10}$ An der Charité wurde das Medikament ebenfalls seit 1959 verabreicht, wenngleich es im ersten Jahr nur 19 Behandlungsfälle gab. ${ }^{11}$ Die Tofraniltherapie von Hans Arnold fällt somit noch in die Erprobungsphase des Medikaments in der DDR.

Das Präparat wurde vermutlich auch aufgrund der Knappheit vergleichsweise selten eingesetzt, wie ich im Folgenden belegen werde. Die Hoffnung auf eine medikamentös induzierte Besserung erfüllte sich im Falle von Hans Arnold jedoch nicht. Am 1. September 1960 vermerkten die Psychiater im Verlaufsbericht: „Status idem. Tofranilkur weiterführen“. 14 Tage später, am 13. September 1960, schien sich der Zustand nicht merklich geändert zu haben. So gab der Patient auf Befragung an „dass es wieder schlechter werde, denn der Schlaf sei schon gar nicht mehr da. Die Frau habe auch während der ganzen Besuchszeit nur auf dem Bett gesessen und geweint. Er könne gar nichts dagegen tun, diese furchtbare Stummheit lasse sich nicht überwinden." Selbst mehr als drei Wochen nach Beginn der Behandlung wurde der Patient als völlig stumm geschildert, obwohl er sich offenbar im Bezug auf seine Beschwerden gut zu artikulieren wusste. Im Verlaufsbericht vermerkte der behandelnde Arzt schließlich: „Das Tofranil scheint ohne jede Wirkung“, was die Vermutung nahelegt, die Ärzte hätten die Therapie aus diesem Grund beendet. Die weitere Lektüre zeigt eine andere Lesart auf, denn im Verlaufsbericht vom 12. Oktober 1960 räumten die Ärzte schließlich ein, dass „die Tofranilkur nicht weiter fortgeführt werden [konnte], weil z. Zt. Tofranil nicht mehr zur Verfügung steht. “12

Den Arzneimittelmangel nahmen die Charitéärzte aber nicht widerspruchslos hin. Wie man den Verwaltungsakten der Nervenklinik entnehmen kann, wurde auf der Sitzung der Verbindungsärzte im Versorgungsausschuss der Charité auf den weiteren Import von Tofranil als unbedingt notwendig gedrungen. Zudem klagte die Klinik „daß 8-9 Monate vergehen bis ein neues Präparat routinemäßig eingesetzt werden“ könne. ${ }^{13}$ Ende 1961 spitzte sich der Mangel an Imipramin weiter zu, da das Ministerium für Gesundheitswesen im Zuge der Störfreimachung den weiteren Import ablehnte und auf die bald zur 
Verfügung stehende ungarische Nachentwicklung verwies. ${ }^{14}$ Dieser Import behob aber erst in der zweiten Jahreshälfte 1963 den Medikamentenengpass an der Charité. ${ }^{15}$

Arnold selbst mögen diese Hintergründe egal gewesen sein. Die zunehmende Verschlechterung seines Zustandes registrierte er jedoch genau. So vermerkt der Verlaufsbericht Mitte Oktober, dass Arnold auf die Frage nach den Erfolgen der Behandlung nur knapp erwiderte: „Nein, es ändert sich nicht mehr." Die Gründe für den Misserfolg der Behandlung suchte der Patient nun in seinem eigenen Leben. Er könne sich aber nicht erklären, warum er so gestraft werde, wo er sein Leben der Arbeit gewidmet habe. Der Chef habe ihn nur ungern mit den Worten, dass nun "der Beste gehe“, aus dem Dienst entlassen. Häufig stelle er sich deshalb die Frage, warum sein Zustand sich so verschlechtert habe, oder wie er es formulierte: „Von was kommt das, dass ich mich mit niemanden mehr unterhalten kann?" Die niedergedrückte Stimmung des Patienten entging den Ärzten nicht, die eine engmaschigere Überwachung veranlassten. ${ }^{16}$ Leonhard hatte in seiner „Einteilung der endogenen Psychosen" auf die besondere Suizidgefahr bei Melancholien hingewiesen (Leonhard 1968: 25). Es wundert also nicht, dass seine Schüler verstärkt auf suizidales Verhalten des Patienten achteten. Als schließlich bemerkt wurde, dass der Patient mit einem Rasierapparat in der Tasche zur Toilette ging, entschlossen sich die Ärzte zur Verlegung auf die geschlossene Abteilung. In einem Brief, den er an seine Frau aus der Klinik schrieb, wird die Verzweiflung des Patienten über diese Zwangsmaßnahme deutlich. Das Krankenblatt vom 21. Oktober 1960 hielt folgende für die Ärzte wichtigen Passagen fest: ${ }^{17}$

Es tut mir weh, wenn andere sich unterhalten und ich still dasitzen muß und ich nicht sprechen kann. Keiner wird mir helfen können, ich glaube nicht an Wunder. Ich bin ein Mensch zweiter Klasse. Das Werk wird mich auch nicht wieder einstellen und wir werden wieder Hunger leiden. Ich quäle mich nun schon Jahre hindurch und Du mit mir. Wer weiß ob ich unseren Garten nochmals wieder sehe. Ich sitze hier nun schon wieder monatelang ohne Hilfe. Es ist mir gestern soviel Leid zugefügt worden wie noch nie in meinem Leben (Stationsverlegung). Ich habe die Lust zum Leben verloren und keine Freude mehr. ${ }^{18}$

Die Klinik verstärkte ihre therapeutischen Bemühungen angesichts der zunehmend trüben Stimmung des Patienten und dem Schwinden seiner Hoffnung. Da die medikamentöse Behandlung nicht anschlug, stellten die Ärzte Anfang Dezember die Diagnose erneut in Frage: „Melancholie? Gehetzte Depression?" Da eine Elektroschockbehandlung aufgrund des schlechten körperlichen Zustands des Patienten als nicht ratsam erschien, erwogen sie eine Umstellung auf das Neuroleptikum Chlorpromazin (DDRHandelsname: Propaphenin).

Chlorpromazin wurde als erstes Mittel gegen Psychosen weltbekannt. ${ }^{19}$ Zwar war die Substanz in den 1950er Jahren zunächst auch als 
Antidepressivum eingesetzt worden, ${ }^{20}$ doch hatte man Anfang der 1960er Jahre ihren Gebrauch auf die antipsychotische Indikation eingegrenzt. ${ }^{21}$ Dass die Psychiater der Charité die depressiven Verstimmungen von Arnold noch Anfang der 1960er Jahre mit Chlorpromazin behandelten, erklärt sich nicht durch einen Mangel an Wissen, sondern durch das Fehlen von Alternativen. Während es im Westen mit Phenelzin und Iproniazid bereits weitere Antidepressiva gab, stand den DDR-Psychiatern neben Imipramin noch 1961 kein weiteres Antidepressivum zur Verfügung. ${ }^{22}$ Es gibt aber auch wissenschaftliche Begründungen für den Wechsel zu einer neuroleptischen Behandlung: Leonhard ordnete sowohl die Melancholie als auch die gehetzte Depression den Psychosen zu. So gilt als Merkmal der Leonhard'schen Lehre, den Kreis der bipolaren Psychosen und psychotischen Depressionen wesentlich größer zu fassen als andere psychopathologische Konzepte. In diesem Sinne war die Kur mit einem „Antipsychotikum“ keineswegs abwegig (Leonhard 2003).

Die Propapheninbehandlung Arnolds wurde mit hohen Dosen begonnen. Auch diese verbesserte den Zustand des Patienten kaum. Ende Dezember erhielt Arnold bereits $400 \mathrm{mg}$ täglich - deutlich mehr als die übliche Dosis: ${ }^{23}$ „Unter dieser Dosierung ist der Pat. fast parkinsonistisch ${ }^{24}$, zeigt sowohl eine mimische als auch allgemeine körperliche Starre, ist sehr klagsam, erscheint äußerst gequält. “25 Als sich zusätzlich noch eine Zyanose ${ }^{26}$ der Schleimhäute ausbildete, die lebensbedrohliche Folgen haben konnte, wurde Propaphenin abgesetzt. Anfang Januar registrierten die Ärzte eine lockerere Körperhaltung des Patienten. Auch gab er selbst an, dass er sich etwas freier fühle. Ob die Therapie nun angeschlagen hatte oder Arnold nur von den beschwerlichen unerwünschten Wirkungen befreit werden wollte, muss offen bleiben. Der kleine Erfolg führte die Ärzte dazu, eine weitere Variante der Propapheninbehandlung anzuwenden: Die Stoßtherapie. Hierfür wurden zunächst hohe Dosen des Propaphenins verabreicht, die dann abrupt abgesetzt wurden.

Die Charité-Psychiater hatten dieses zunächst ungewöhnliche Regime nicht selbst entwickelt. Bereits 1959 publizierte der westdeutsche Psychiater H. Neumann ${ }^{27}$ in der Zeitschrift Der Nervenarzt über die Erfolge dieser „Zackenbehandlung“. Ihr Vorteil sei, wie Neumann herausarbeitete, dass „chemische[n] Vergiftungen“ nicht so häufig aufträten wie bei der Dauermedikation (Neumann 1959: 253). Es ist davon auszugehen, dass diese Beobachtung auch für die Ostberliner Psychiater leitend war, da die hochdosierte Dauereinnahme bei ihrem Patienten zu schweren Komplikationen geführt hatte. Nach Neumann galt als besonderer Vorteil der Stoßtherapie, dass die Kranken sich zwischen den einzelnen Therapiephasen frei bewegen und beispielsweise ohne Hilfe die Toilette aufsuchen könnten (Neumann 1959: 254). So fand die Zackenbehandlung in manche Klinik der DDR Eingang ins Behandlungsrepertoire. Horst Berthold (1924-1969), Leiter der Bezirksnervenklinik Schwerin, bestätigte in einem Überblicksartikel die Erfolge der in den 
letzten Jahren an seiner Klinik durchgeführten Zackenbehandlungen (Berthold 1963: 305).

Anfang 1961 startete man an der Charité mit dem neuen Verfahren: Am 8. Februar wurde im Krankenblatt vermerkt: „Kurzfristige Steigerung des Propaphenin auf $400 \mathrm{mg}$ und schlagartiges Absetzen. Unter dieser Therapie lockert sich das psychische Verhalten etwas auf, jedoch ohne eine deutliche Besserung. ${ }^{\text {28 }}$ Die Stoßtherapie wurde insgesamt dreimal wiederholt und Ende März beendet.

Zunächst beurteilten die Ärzte Arnold weiterhin als depressiv. Die Ehefrau, die inzwischen wegen des mangelnden Therapieerfolges ungeduldig geworden war, drohte nun damit, den Ehemann gegen ärztlichen Rat aus der Klinik zu nehmen, da er ihrer Meinung nach „hier kränker geworden wäre als zuhause“. Der Patient hingegen erklärte sich zu einem weiteren Propapheninstoß bereit, der den Berichten der Klinik zufolge dazu führte, dass der Patient etwas lockerer sei und nicht mehr über Sprachhemmungen klage. Der Erfolg hielt jedoch nicht lange an. Von kurzen Propapheninkuren abgesehen verzichteten die behandelnden Ärzte in den nächsten Monaten weitgehend auf die Verordnung von Psychopharmaka.

Es sind aber auch andere Gründe für den anhaltend melancholischen Zustand denkbar. Kurz vor der Einleitung der "Zackenbehandlung“ war Arnold von dem Besuch eines Vertreters seines alten Arbeitgebers überrascht worden, der ihm einen Aufhebungsvertrag offerierte. Aus der Sicht der Klinik hätte der Aufhebungsvertrag dem Patienten nur Vorteile geboten. Aus der Sicht des Patienten, der sich stark mit seiner Arbeit identifizierte, kam dieses Angebot jedoch einer massiven Entwertung gleich. So wurde über das Gespräch des Patienten mit dem Vertreter des Werkes notiert:

Er glaubt, dass man ihn hinauswerfen wolle [...]. Während der Besprechung sitzt er zusammengekauert auf dem Stuhl, weint haltlos vor sich hin, stützt den Kopf in die Hand, ist ratlos und geht auf keinen Vorschlag ein. ${ }^{29}$

Es lässt sich nur spekulieren, ob die Verzweiflung über den drohenden Verlust der Arbeit einen weiteren Behandlungserfolg verhinderte. Am 24. August 1961, fast fünf Monate nach der letzten Behandlung, hielten die Psychiater im Verlaufsbericht fest, dass sich das Zustandsbild des Patienten nicht geändert hätte. Deshalb wurde jetzt eine Kur mit Iproniazid (Handelsname: Marsilid) erwogen, ein Arzneimittel, das in der DDR als Import nicht für die allgemeine klinische Anwendung zur Verfügung stand.

Nathan Kline (1916-1983), der die Effekte des MAO-Hemmers (Monoaminooxidase-Hemmer) 1957 und 1958 beschrieben hatte, hielt Iproniazid für ein gutes Antidepressivum, weil es euphorisierend wirke (Loomer/Saunders/ Kline 1957).$^{30}$ Seit 1961 wurde die Substanz jedoch wegen ihrer lebertoxischen Wirkung nicht mehr als Antidepressivum in den angloamerikanischen Ländern eingesetzt (Maxwell/Eckhardt 1990: 455). Wie man den Protokollen des 
für die Einführung von neuen Pharmaka verantwortlichen Zentralen Gutachterausschusses beim Ministerium für Gesundheitswesen (ZGA) entnehmen kann, wurde schon 1960 die Anfrage zur Produktion von Iproniazid an den Ausschuss herangetragen, die Nachentwicklung aber zurückgestellt. ${ }^{31}$ Auch in den folgenden Jahren fiel diese Entscheidung nicht vermutlich aufgrund der aufkommenden internationalen Kritik am Einsatz von Iproniazid.

In der DDR blieb die Zurückstellung jedoch nicht ohne Widerspruch, zumal es neben Imipramin kein anderes Antidepressivum gab. So drängte der Leipziger Ordinarius Dietfried Müller-Hegemann (1910-1989) auf eine reguläre Einführung von Iproniazid auch in der DDR. Seine Klinik habe mit Iproniazid gute Erfahrungen gemacht, ließ er das Ministerium wissen. Bereits 1961 hätten die VEB Leuna Werke auf Wunsch der Klinik größere Mengen an „Marsilid“32 zu Erprobungszwecken hergestellt, ihre Produktion aber danach einstellen müssen. Wie Müller-Hegemann zuspitzte,

ergibt sich die Situation, dass wir in der DDR kein Mittel zur Verfügung haben, um schwere Depressionen im Sinne eines Thymolepticums gezielt zu behandeln. Im Zusammenhang mit der notwendigen "Störfreimachung“ wäre dann allerdings zu bedenken, dass wir auf Importe angewiesen sind, die im Großen und Ganzen aus dem westlichen Ausland erfolgen müssten. ${ }^{33}$

Auch an der Charité führte diese Politik der „Störfreimachung“ zu eklatanten Versorgungsengpässen. Auch wenn wir den genauen Weg nicht kennen, so ist doch davon auszugehen, dass die Nervenklinik ebenfalls eine Marge des von den Leunawerken versuchsweise hergestellten MAO-Hemmers erhielt. Im offiziellen Versorgungsausschuss der Charité wurde das Westprodukt mit keinem Wort erwähnt. Da die Nervenklinik aber nachdrücklich dazu angehalten war, die umfangreiche Wunschliste von Importmedikamenten aufgrund des knapp bemessenen Budgets der Klinik und eingeschränkter Importmittel noch einmal zu überprüfen, ist anzunehmen, dass sie keine weiteren Importwünsche zu äußern wagte. ${ }^{34}$

Hans Arnolds Therapie mit „Marsilid“ begann schließlich wenige Tage nach dem Mauerbau. Mitte September hielt die Krankenakte fest, dass man mit dem Patienten gut in Kontakt komme. Schon eine Woche später relativierten die Psychiater diese Besserung:

Es ist sicher im Vergleich zu den früheren Beobachtungen jetzt eine gewisse Auflockerung und Aktivierung bei dem Pat. festzustellen. Im Großen und Ganzen jedoch hat sich wohl an dem Gesamtzustand nicht viel verändert. ${ }^{35}$

Angesichts der ausbleibenden Erfolge so unterschiedlicher therapeutischer Ansätze stellten die behandelnden Ärzte ihre Diagnose nochmals in Frage. Sie veranlassten eine erneute Röntgenübersichtsaufnahme des Thorax, in der sich eine ausgeprägte Aortensklerose zeigte, ${ }^{36}$ was auch heute noch unter Psychiatern als Hinweis auf einen altersbedingten Hirnabbau mit schlechter 
Prognose gilt. Neben der Diagnose vermerkte der behandelnde Arzt mit Bleistift „Therapieresistent!“. Diese Einschätzung, dass sie ihn für wenig behandelbar hielten, teilten die Ärzte Arnold jedoch nicht mit. Sie führten trotz allem eine weitere Marsilidkur durch, die wiederum keinen klinisch evidenten Therapierfolge brachte. Auch Arnold entging nicht, dass sich sein Zustand keinesfalls besserte. Auf sein Befinden angesprochen bemerkte er: „Alles ist tot". Der Patient erwartete sich von den Ärzten jedoch auch weiterhin umfassende Hilfe und sprach sie häufig auf eine bessere Behandlung an, was diese jedoch als Teil der pathologischen Symptomatik disqualifizierten:

[Er] hält den Arzt durch unaufhörliches Besprechen immer derselben Angelegenheit auf. Sobald der visitierende Arzt glaubt, das abschließende Wort gesagt zu haben, beginnt der Patient von neuem mit dem gleichen Thema. Spricht dann auch den Arzt immer wieder auf dem Flur an, kommt mit seinem Denken nicht von der Stelle. ${ }^{37}$

Mitte März 1962 wurde Arnold schließlich aus der Klinik entlassen.

Betrachtet man die geschilderte Behandlung mit dem Wissen um die vorhandenen Psychopharmaka der Zeit und die in der Charité üblichen Therapien wird deutlich, dass Arnold scheinbar bevorzugt jene Medikamente verabreicht bekam, die in der DDR nur äußerst knapp zur Verfügung standen. So gehörte Arnold zu der kleinen Gruppe von Patienten, die mit Imipramin (Tofranil) behandelt wurden. Und mehr noch: die Ärzte unternahmen darüber hinaus einen Behandlungsversuch mit dem in der DDR nur zu Versuchszwecken hergestellten Iproniazid (Marsilid). Obwohl Arnold im Rahmen der Klinik im Wesentlichen als selbstbewusster Patient agierte, zeigen sich hier schon einige Merkmale, die für seinen Konsumentenstandpunkt charakteristisch werden sollten: der unbedingte Glaube daran, dass sich sein Zustand durch weitere Hilfe verbessern lasse, sowie seine Identifizierung mit seiner Stellung als Arbeiter in einem Arbeiter- und Bauernstaat. Wie ich zeigen werde, nutze Arnold beide Positionen im weiteren Verlauf eindrucksvoll, um weitere Medikamente zu erhalten.

\section{„Wo doch mein ganzes Leben nur Arbeit für den Staat und die Gesellschaft war"'. Arnolds Blick auf sich selbst und sein Glaube an das sozialistische Gesundheitswesen}

Ein Jahr nach seiner Entlassung meldete sich Arnold erneut. In einem langen Brief beklagte er seinen weiterhin schlechten Zustand und bat um effektive Medikamente: Wie er ausführte, habe er selbst

alles versucht meine schwere seelische Krankheit die auch sehr viel durch Ärger mit schwerer Überanstrengung entstanden ist zu verbessern. Bis jetzt geht es mir aber noch nicht wesentlich besser [...]. Aus dem Stahlwerk Riesa was ich 1946 als 
erster wieder aufgebaut hab haben sie mich ganz abgeschrieben [...] Wie und was kann ich allein den überhaupt noch tun, um meinen Zustand zu verbessern. Welche Medikamente helfen denn da überhaupt? Ich wende mich darum wieder mit der Bitte an Sie [Prof. Leonhard], da Sie ja mit ihren großen Erfahrungen bekannt im In- und Ausland sind. Hoffe, das Sie mir nun eine gute Nachricht [senden] und etwas verordnen, das mir wieder hilft, meine Krankheit zu heilen. Damit ich wieder am gesellschaftlichen Leben in der DDR mitmachen kann. ${ }^{38}$

Arnolds Brief zeugt, trotz der ausgebliebenen Erfolge der bisherigen Behandlung, von einem ungebrochenen Glauben an die therapeutischen Möglichkeiten. Dass sie seinen Zustand aus medizinischer Sicht nicht mehr grundsätzlich für therapierbar hielten, hatten die Psychiater ihm entweder noch nicht mitgeteilt oder Arnold wollte sich mit dieser Erklärung nicht abfinden. Ebenso wenig hatte Arnold offenbar realisiert, dass die Ärzte seinen harten Arbeitseinsatz für den Wiederaufbau des sozialistischen Produktionswesens, als einen wesentlichen Faktor seiner Erkrankung betrachteten. Dabei sei dahingestellt, ob die Ärzte den depressiven Zustand tatsächlich auf übermäßige Arbeit zurückführten oder ob sie lediglich Arnolds große Heilungserwartung dämpfen wollten. Mit umsichtig gewählten Worten versuchte ein Oberarzt der Klinik in einem Antwortschreiben Arnold seine schwierige Situation zu erklären:

Sie wissen, lieber Herr [Arnold], dass wir sehr gerne alles tun würden, Ihnen zu helfen. Sie wissen aber auch und wir wollen es Ihnen nicht verheimlichen, dass es sich bei Ihnen zum großen Teil um vorzeitige Alters- und Abnutzungserscheinungen handelt. Sie haben in ihrem Leben sehr viel geleistet, aber dabei leider auch sehr viel von ihrer Gesundheit hergegeben. Es wäre schon sehr viel wert, wenn Sie wieder in einen für Sie erträglichen Gesundheitszustand kommen. Sie sollten nicht den Ehrgeiz haben, selbst weitere aktive Leistungen zu vollbringen. ${ }^{39}$

Tatsächlich war diese Antwort heikel, da sie den als Leitlinien propagierten Zielen der sozialistischen Staatsführung nicht entsprach. Seit der Staatsgründung wurde immer wieder offiziell betont, es sei ein wesentlicher Vorzug einer sozialistischen Gesellschaft, dass sich der Arbeiter im Produktionsprozess entfalten und seine Persönlichkeit in der Erwerbsarbeit voll entwickeln könne. Diese Bedeutung der Arbeit zeigt sich auch in der Vielzahl von Auszeichnungen wie beispielsweise „Held der Arbeit", die materielle und gesellschaftliche Anerkennung brachten. Die Idealisierung der Arbeit korrespondierte aber auch mit den gesellschaftlichen Erfordernissen einer DDR, die den Wiederaufbau nur durch einen immensen Einsatz menschlicher Arbeitskraft bewältigte (Bauerkämper 2005: 12). Diese Staatslehre sah nicht vor, dass Überarbeitung zu psychischen Krankheiten führte. Solche Leiden galten Anfang der 1960er Jahre als „Managerkrankheiten“ in jenem kapitalistischen System deren Gesellschaftsstruktur die DDR doch gerade überwinden wollte. So wurde beispielsweise die Nachentwicklung der in den westlichen Staaten seit den 1950er Jahren vertriebenen Tranquilizern vom Zentralen Gutachterausschuss abgelehnt, weil das Gremium keine gesellschaftliche Notwendigkeit 
für die Einführung eines Medikaments gegen arbeitsbedingte Angst- und Erschöpfungszustände sah (Klöppel/Balz 2010). Hans Arnold, bekennender Sozialist der ersten Stunde, wollte diese vorsichtigen Hinweise auf die Ursachen seines Leidens zunächst nicht hören. Zu sehr hatte sich seine eigene Identität mit seinen Aufbauleistungen im Betrieb verwoben. In einem Antwortbrief vom 20. Mai 1963 hob er auf diesen für ihn entscheidenden Punkt ab:

Der liebe Gott hilft mir nicht, da ich keine Kirche besucht hab. Ich hab ja in meinem Leben nie Zeit gehabt, da ich nur Arbeit kannte. [...] Bei uns im Werk gab es jede Woche kurze Schichtwechsel. Man hatte nur vier Stunden Zeit zum Schlaf. Ich schlief natürlich die wenigen Stunden nicht, da ich schon wieder gerechnet hatte wie weit die Arbeit sein konnte wenn ich die Arbeit wieder antrat. Weiter war ich zweimal Aktivist ${ }^{40}$ und zweimal Bestarbeiter noch dazu Parteigenosse. Das hieß für mich gesellschaftlich und in der Arbeit Vorbild sein. Ich habe weil Leutemangel war auch meinen Urlaub meistens geteilt genommen, bin nie in ein Ferienheim oder zu einer Erholungskur gefahren. Meine Maschine war mein alles. Sie habe ich gepflegt und geachtet mehr wie meine gute Frau. Der habe ich jetzt verraten müsse das ich die Uhr oft vorgedreht hab damit ich schon eine Stunde zuvor Schichtwechsel [...] im Betrieb war. Heute lachen mich meine Kumpel aus und auch mit Recht da ich nun zuhause sitze und hoffe, dass mir nun jemand hilft, daß ich wieder gesund werde. Habe auch oft meine Prämien verteilt, die ich für gute Leistungen bekam. Als ich dann krank wurde, hat mich ein verdienter Arzt des Volkes, der sein Krankenhaus dann verlassen hat und jetzt im goldenen Westen sitzt mich von meiner Arbeit weggenommen, die ich immer zur Zufriedenheit meiner Kumpel gemacht hatte. [...] Es setzte bei mir der Ärger ein. ${ }^{41}$

Für die Opfer, die er dem Sozialismus gebracht hat, erwartete Arnold nun eine adäquate Gegenleistung. Er selber nahm jede Möglichkeit wahr, sich über seine Krankheit zu informieren. Wie er in dem Brief berichtete, schaute er sich beispielweise nahezu täglich bei einer befreundeten Familie, die einen Fernsehapparat besitze, alle gesundheitsaufklärenden Sendungen an. Nie würde dort aber über seine Krankheit gesprochen. Auch sein Hausarzt habe ihm gesagt, dass er ihm nicht helfen könne. So schloss Arnold seinen Brief an den Oberarzt der Charité abermals mit der Bitte: „Helfen sie mir mit Rat und Medikamenten, damit ich wieder etwas Freude im Leben habe, denn ich habe ja nun im Leben meine Pflicht getan. “42 Seiner Bitte entsprach man nicht. Der Oberarzt der Charité versicherte, dass der Klinik keine Verordnung ohne vorangegangene Untersuchung erlaubt sei. Zudem könne er nichts anderes verordnen als jeder Hausarzt, der ebenfalls alle in der DDR erhältlichen Medikamente verschreiben könne. Diese Antwort befriedigte Arnold jedoch keineswegs. Sie war vielmehr Anlass dafür, dass er sich nun auf eigene Faust auf die Suche nach neuen Psychopharmaka begab. 


\section{„Da habe ich mich an die Sowjetunion gewandt” - Arnolds grenzüberschreitende Aktivitäten}

Drei Monate später, im Juni 1963 schrieb Arnold erneut an die Nervenklinik der Charité: „Seit 1957“ so begann der ehemalige Patient seinen Brief, „habe ich schon diese schwere Depression und habe oder hatte ich keine Selbstmordgedanken, auch in Berlin nicht. Aber auf den Friedhof habe ich mich schon oft gewünscht weil es in unserem Arbeiter- und Bauernstaat auch keine Mittel gibt um solche Krankheiten zu heilen nur zu trösten wie Sie es taten als ich von Berlin krank fort ging."

Die Klage über enttäuschend unzureichende Hilfe angesichts gesellschaftlicher und politischer Leistungen schloss Arnold aber mit dem Rapport seiner Eigeninitiative. Weil sich die Ärzte der Charité als insuffizient erwiesen hätten, habe er sich

in meiner Verzweiflung auch an das Ministerium für Gesundheitswesen in Moskau gewandt mit der Bitte, mir zu helfen, da ich im faschistischen Raubkrieg verschleppte russische Kriegsgefangene unterstützt hab. Habe das Schriftstück hier in meinem Besitz. Sollten Sie Interesse noch an mich und dem Schriftstück haben so bin ich gerne bereit, es Ihnen zu schicken damit ich vielleicht von diesem Freunde etwas bekomme, was mir das Leben erträglich macht. Damit bei der guten Frau und den Kindern die Tränen in den Augen recht bald verschwinden, denn Sie wissen das mein ganzes Leben nur Arbeit für den Staat und der Gesellschaft war und ich jetzt dahinsiechen muß vergessen und verlassen. ${ }^{43}$

Die Ärzte der Charité sehen sich jedoch nicht veranlasst, der Antwort ihrer sowjetischen Kollegen vorzugreifen, wohl wissend, dass die Psychiater dort auch nicht über andere Medikamente verfügten. Seit Bestehen der DDR wurden in der einzigen psychiatrischen Fachzeitschrift Psychiatrie, Neurologie und medizinische Psychologie immer wieder Aufsätze von Kollegen aus sozialistischen Ländern zum Thema Psychopharmaka veröffentlicht. Der Stand zu somatischen Behandlung von Depressionen in der Sowjetunion war den ostdeutschen Psychiatern darüber hinaus durch die Zusammenfassung eines thematisch einschlägigen Kongresses in der Sowjetunion bekannt. ${ }^{44}$ Erwartungsgemäß erhielt Arnold, wie er die Charité wissen ließ, auch vom "großen Bruder" diese Auskunft. So schloss er einen erneuten Brief, dem er das Schreiben der sowjetischen Psychiater beifügte mit der Bitte, „mir solche zu verordnen oder mir zu schreiben, dass es nichts gibt, damit ich es an die Sowjetunion schicken kann. ${ }^{45}$ Daraufhin entgegnete die Klinik wunschgemäß, dass die von den sowjetischen Kollegen aufgelisteten Medikamente genau jenen entsprächen, die der Patient auch in der Nervenklinik bekommen habe und die empfohlene Behandlung ohne weiteres vom Hausarzt seines Wohnortes durchgeführt werden könne. ${ }^{46}$

Arnold war inzwischen nicht untätig geblieben. Er habe gehört, so beklagte er sich im folgenden Monat, dass die Charité dem Hilferuf polnischer 
Kollegen gefolgt sei und ein wichtiges Medikament für schwer erkrankte Kinder sofort zur Verfügung gestellt habe. Er bitte noch einmal darum, ihm ebenfalls ein hilfreiches Medikament $\mathrm{zu}$ senden. ${ }^{47}$ In einem folgenden Schreiben ließ er die Charité-Ärzte wissen, er habe sich nun auch an andere Ordinarien wie Dr. Hanns Schwarz (Greifswald, 1898-1977) sowie an das Gesundheitsministerium der DDR gewandt, die ihm aber ebenfalls nicht geholfen hätten. ${ }^{48}$ Welche Wellen Arnolds Aktionismus schlug, wurde den Berliner Psychiatern aber erst durch einen brieflichen Hinweis des behandelnden Hausarztes vor Augen geführt. „Sie wissen vielleicht“, so formulierte dieser vorsichtig, „dass er [Arnold] über den Äther mit vielen Rundfunkstationen der Welt in Verbindung steht und versucht, irgendwoher Hilfe zu erhalten. Er ist der Meinung, daß es bei der fortschreitenden Wissenschaft doch möglich sein sollte, für ihn ein Präparat zu finden, das ihm seine Arbeitsfähigkeit wiedergibt." 49

Auch diese eigenmächtigen Handlungen waren von offiziellen Versprechungen der Parteispitze motiviert, hatte diese doch verkündet, der wissenschaftlich-technische Fortschritt werde das Gesundheitswesen stetig verbessern. Dieser Fortschrittsoptimismus fand seinen Höhepunkt in dem 1967 vom Rat für Planung und Koordinierung der medizinischen Wissenschaften organisierten Kongress, der unter dem Titel „Sozialismus, wissenschaftlich-technische Revolution und Medizin“ über die Erfolge der modernen Medizin berichtete. Gerade die technische Revolution, so hieß es dort, werde zu entscheidenden Verbesserungen der Medizin führen, wenn sich diese ganz in den Dienst des Sozialismus stelle (Gudowski/Wissenschaft 1968).

Diese Zukunftsversprechungen standen im krassen Gegensatz zur Gegenwart der psychiatrischen Praxis. Vor allem in den Landeskliniken war die Versorgung stark eingeschränkt. So gestand der bereits erwähnte Hausarzt Arnolds, dass er Leichtkranke keinesfalls in eine Klinik vor Ort einweise, da diese in den ihm bekannten Fällen in einem schlechteren Zustand zurückgekehrt seien. Wegen des Ärztemangels werde in den psychiatrischen Landeskrankenhäusern keine richtige Behandlung durchgeführt. ${ }^{50}$ In der Tat war die Situation in diesen Einrichtungen aufgrund der bis zum Mauerbau eklatanten Ärzteflucht, des Bausubstanzverfalls und mangelnder therapeutischer Möglichkeiten ausgesprochen schwierig, wie einige Studien gezeigt haben. ${ }^{51}$ Auch die Bemühungen um eine grundlegende Reform der psychiatrischen Verwahrpraxis und die Etablierung einer auf Rehabilitation gerichteten Behandlung stagnierten weitgehend trotz des seit 1959 vom Ministerium für Gesundheitswesen eingesetzten Fachausschusses für Psychiatrie. So räumte der verantwortliche Vorsitzende des Fachausschusses, Ehrig Lange (1921-2009), in einer Rede vor Ministerialvertretern mit Blick auf die Verantwortung des Ministeriums für Gesundheitswesens ein, dass „der entscheidende Durchbruch aber, aus der gesellschaftlichen Bedeutung heraus die 
psychiatrischen Entwicklungsaufgaben zu Schwerpunktaufgaben in Forschungs- und Entwicklungspolitik zu transportieren, [...] ökonomische und finanzielle Kapazitäten“ erfordere, die man den Einrichtungen „nicht in ausreichendem Maße zur Verfügung gestellt" habe. ${ }^{52}$

Auch Arnold schien die eingeschränkten psychiatrischen Hilfsmöglichkeiten in der DDR inzwischen wahrgenommen zu haben. Seine nunmehr zwei Jahre andauernden Anstrengungen um eine medikamentöse Besserung seiner Beschwerden hatten wenig Erfolg gezeigt. Nun wandte er sich hilfesuchend an das kapitalistische Ausland. In einem Brief vom 11. November 1963 teilte Arnold der Charité mit, dass er zwischenzeitlich Besuch aus Westdeutschland erhalten habe, der ihm ein neues Medikament von einem dort tätigen Arzt mitgebracht habe. Um die Behandlung fortzusetzen, benötige er jedoch nun ein Rezept von einem ihn behandelnden Arzt der DDR, da die Versendung des Arzneimittels aus Westdeutschland von den DDR-Grenzbeamten streng kontrolliert würde. ${ }^{53}$ Westgeschenke waren nach Merkel ein entscheidender Faktor für die Konsumkultur in der DDR. Es handelte sich aber nicht um kriminelle Handlungen, solange sich Sender und Empfänger an die rigiden Zollbestimmungen hielten. Dennoch wurde der Paketverkehr genau beobachtet und kontrolliert (Merkel 1999: 289). Anders verhielt es sich mit Geschenksendungen rezeptpflichtiger Arzneimitteln. Diese waren zu diesem Zeitpunkt bereits verboten. ${ }^{54}$ Wie das Schreiben eines Abteilungsleiters des Ministeriums für Gesundheitsministerium von 1961 ausführte, befürchtete man offenbar, dass die pharmazeutische Industrie des kapitalistischen Auslandes durch die systematische Versendung solcher Muster einen künstlichen Bedarf schuf. ${ }^{55}$ Dass die Annahme solcher Geschenksendungen ein ernsthaftes Vergehen sei, zeigt die Antwort der Charité: „Im übrigen ist es verboten, Rezepte für westdeutsche Präparate auszustellen. ${ }^{\text {"56 }}$ Mit deutlichen Worten versuchte der Arzt nun, weitere Aktivitäten Arnolds zu unterbinden.

Es gibt keine andere Behandlung, die mehr erreichen könnte, weder bei uns noch in der Sowjetunion, noch in Westdeutschland. Sie sollten in Zukunft unbedingt davon absehen, sich an alle möglichen Stellen zu wenden. ${ }^{57}$

Zwar sei er grundsätzlich bereit, ihn zu unterstützen, wie der Arzt weiter ausführte, doch habe es keinen Sinn, die immer gleichen Fragen zu wiederholen. Eine wesentliche Besserung seines Zustandes könne nur eintreten, wenn er sich nicht beständig mit seiner Krankheit beschäftige. Dass aus der BRD erhaltene Medikament, so der Oberarzt weiter, weise keine Vorteile gegenüber den in der DDR vorhandenen auf.

Hans Arnold erboste die Antwort des Oberarztes. Der Arzt bringe, wie Arnold in seinem Antwortschreiben hervorhob, damit zum Ausdruck, es würde sich bei seiner Krankheit um einen unheilbaren Zustand handeln, dem man keine therapeutischen Bemühungen mehr schenke. Er zähle aber „immer noch zu den Lebenden, wenn auch schwer krank, und bin auch ein Bürger der 
DDR der Recht auf alles hat, um seine Krankheit $\mathrm{zu}$ verbessern und $\mathrm{zu}$ heilen." ${ }^{58}$

Arnold hatte durch die selbstbewusste Forderung nach besserem Medikamentenkonsum seinen eigenen Gesundheitszustand bisher zwar nicht verbessern können, gleichzeitig hatte er aber gezeigt, dass er keine Mühen scheute, sich aktiv an der Auswahl effektiver Medikamente zu beteiligen. Dabei verließ er sich nicht mehr auf die Auskünfte, die er in der DDR erhielt sondern überprüfte diese kritisch, indem er unabhängige Informationen aus der Sowjetunion und sogar aus dem kapitalistischen Ausland einholte. Das (imaginierte) hilfreiche Medikament zu erhalten, gelang ihm jedoch nicht, sodass er nach neuen Wegen suchen musste. Es sollten drei Jahre vergehen, bis der Oberarzt wieder Post erhielt. Diesmal bemühte Arnold die Partei für sein Anliegen.

\section{„Der Kreissekretär möchte wissen, was sie mir verordnet haben“ - Hans Arnold als Netzwerker}

Ende November 1966 meldete sich Arnold wieder bei den Ärzten der Nervenklinik: Am Beginn des Monats, so der ehemalige Patient, sei seine vierzigjährige Mitgliedschaft in der Arbeiterpartei gefeiert worden. Der Kreissekretär habe ihn zu einer Autofahrt eingeladen und intensiv nach seinem Gesundheitszustand befragt. Da er krankheitsbedingt aber kaum habe sprechen können, habe er ihm schlicht die Briefe des Oberarztes gezeigt, in denen deutlich geworden sei, dass auch die Psychiater der Charité nicht wüssten, woher die Krankheit genau komme. Der Kreissekretär habe sich jedoch nicht damit zufrieden gegeben. Durch Austausch mit den Kollegen im Ausland komme es in der DDR, wie der Funktionär ihm versichert habe, doch zu einer ständigen Weiterentwicklung des Medikamentensortiments. Die Ärzte sollten ihm die neusten Entwicklungen aufschreiben, damit er dem Kreissekretär berichten könne, was er von dort bekommen habe. Diese Information, so Hans Arnold, habe ihn dazu veranlasst, erneut um wirksame Medikamente zu bitten. Am Ende des Briefes fügte er konkretisierend hinzu: „Es muß fürs Schlafen, für den Magen und [für die] Nerven sein.“59

Mit Blick auf die Entwicklung der Psychopharmakotherapie im Jahr 1966 traf die Vermutung des Kreissekretärs, es gäbe neue Medikamente, zumindest auf die Antidepressiva nicht zu. Lediglich der MAO-Hemmer Phenelzin war 1963 in das Arzneimittelverzeichnis der DDR aufgenommen worden. ${ }^{60}$ Die postwendende, negative Antwort des Oberarztes der Charité traf also im Wesentlichen zu. ${ }^{61}$ Arnold gab die Hoffnung aber nicht auf. Mehrfach wandte er sich im nächsten Jahr erneut an die Charité und bat, ihm seine Krankengeschichte zu schicken, damit ein Kollege vom Freien Deutschen 
Gewerkschaftsbund (FDGB) „ihn der SED-Parteileitung vorlegen“ könne: Denn dann „wollen [sie] sich an eine Stelle in Berlin wenden. “62 Die lang ersehnte Erholungskur in einem Heim des FDGB habe er bedauerlicherweise nicht bekommen, weil sein Gesundheitszustand dies nicht zulasse. ${ }^{63}$

Auf den ersten Blick scheint die Mobilisierung einzelner Parteikader nur die weitere Eskalationsstufe einer Patientenklage einzuleiten. Doch diese Einschätzung verkennt den damit einhergehenden Wandel der Subjektposition. Der Wunsch nach einem besseren Arzneimittels wurde nicht mehr von einem psychisch Kranken artikuliert, sondern zum Anliegen einer gesellschaftlichen Gruppe, die Arnolds Hilfsersuchen als eine legitime politische und gesellschaftliche Forderung begriff. Mehr noch: Das Einschalten der Partei mithilfe des Kollegen vom FDGB verwandelte das individuelle Anliegen Arnolds in eine öffentliche Angelegenheit-wenngleich er sie nicht, wie in der bisherigen Konsumentengeschichte beschrieben, mithilfe einer Patientengruppe sondern mit Unterstützung von Parteikadern vertrat.

In der Tat reichte Arnold, vermutlich mit Unterstützung des Kreissekretärs, eine Eingabe an das Ministerium für Gesundheitswesen ein. Seiner Leistungen für den Sozialismus wegen bat er um ein effektives Medikament. ${ }^{64}$ Doch auch diese Eingabe wurde abschlägig beschieden. Wie der zuständige Kreisarzt ausführte, habe eine Behandlung wenig Erfolg, „da es sich um eine Degeneration im Hirnbereich handelt". ${ }^{65}$ Mehr noch: Der vielen Aktivitäten Arnolds wegen habe man in seinem Bezirk „schon mancherlei bereinigen müssen“, klagte der zuständige Kreisarzt. ${ }^{66}$

Auch wenn er das imaginierte Heilmittel nie erhielt, hatte Arnold einiges bewegt: Über Jahre hielt er die (in ihrem Selbstverständnis) bedeutendste psychiatrische Klinik der DDR auf Trab, beschäftigte Psychiater im In- und Ausland, mobilisierte Parteifunktionäre und das Ministerium - immer mit der Bitte seine gesellschaftspolitisch nie bezweifelten Ansprüche zu befriedigen. Dabei nahm Arnold die von Politik, Medizin und Wissenschaft verbreiteten Fortschrittsversprechen ernst. In diesem Sinne erfüllte Arnold die Erwartungen an ein politisch engagiertes sozialistisches Subjekt - und bestand auf der Erfüllung jener Ansprüche, die ihm die sozialistische Gesellschaftsordnung zu versprechen vorgab. Dass er dabei als aktives, grenzüberschreitendes Subjekt agierte, zeigt nur, dass er als selbstbewusster Konsument die bestmögliche Behandlung für sich einforderte - eine Forderung, die für ihn im Einklang mit seiner sozialistischen Grundüberzeugung stand. Im Februar 1969 wandte sich Arnold ein letztes Mal an den Oberarzt der Charité: „Ich weiß nicht, ob Sie in Berlin sind oder ob sie schon in Rente sind," hob sein Schreiben an:

Ich bin nun fast 68 Jahre alt, aber es geht mir sehr schlecht. Merken kann ich mir fast nichts mehr. Liege nun schon so viele Jahre. Laufen kann ich nicht mehr. Meine Frau ist auch schwer krank [...]. Sind nun auf die Stubennachbarn angewiesen. $^{67}$ 
Wie der ehemalige Patient schilderte, seien zu seinem 50. Gewerkschaftsjubiläum zwar noch Kollegen gekommen, er habe sich mit diesen aber nicht mehr unterhalten können. Er bitte ihn doch sehr herzlich, falls diese erhältlich sein sollten, neue Medikamente zu schicken. „Das was ich schreibe, war vorgeschrieben“, beendete Arnold diesen Brief, „habe 2 Stunden gebraucht, denn die Finger haben auch keine Kräfte mehr. “68 In einem Antwortbrief wies der Oberarzt ihn darauf hin, dass er tatsächlich gar nicht mehr in der dortigen Psychiatrie arbeite, sondern andere Aufgaben in der Klinik übernommen habe. Neue Importmedikamente gäbe es aber seines Wissens nach nicht. Doch habe er ihn nicht ohne Antwort lassen wollen, damit der Patient sehe, dass kein böser Wille vorliege. ${ }^{69}$

War es der Rückzug des ihm vertrauten Oberarztes, der Arnold zum Verstummen brachte? Oder hatte sich sein Gesundheitszustand so verschlechtert, dass es ihm nicht mehr gelang, weitere Briefe zu verfassen? Die Gründe für das Ende des inzwischen über sieben Jahre fortgeführten Briefwechsels bleiben offen. Doch bis zum Schluss gab Arnold seine Hoffnung auf eine medikamentöse Heilung nicht auf.

\section{Der Fall Hans Arnolds: Patienten- und/oder Konsumentengeschichte?}

Fallgeschichten, wie sie in Krankenakten zu finden sind, erlauben die Rekonstruktion der kulturellen, politischen und alltagsweltlichen Widersprüche im Sozialismus. Wie Greg Eghigian anschaulich zeigt, spiegelt die von ihm untersuchte Patientengeschichte sehr schön die realen Widersprüche des Kalten Krieges im Wahnerleben einer Grenzgängerin zwischen Ost- und Westberlin wider. Diese Patientin versah diese Erfahrungen, wie Eghigian es formuliert, lediglich mit einem eigenen, der übrigen Welt verdreht erscheinenden Sinn (Eghigian 2003).

Arnolds Eigensinn bestand vor allem darin, dass er auf der Erfüllung der gesellschaftlichen und politischen Heilsversprechungen bestand. Obwohl Arnold schreibend sowohl politische als auch kulturelle Grenzen überschritt, nahm er jedoch die Institutionen des DDR-Gesundheitswesens und der befreundeten Ostblockstaaten in die Pflicht. Gerade in dieser Wendung sprengt Arnolds Geschichte den Rahmen patientengeschichtlicher Ansätze. Arnold verharrte eben nicht in der Rolle des institutionalisierten Subjekts, das sich der Disziplin der Einrichtung und der wissenschaftlichen Neugier der Ärzte fügte. Zwar bezog sich Arnold in allen seinen Aktivitäten auf das medizinische System und folgte den vom System vorgegebenen Bahnen. Doch gleichzeitig überschritt er diese, da er sich nicht als passiver Rezipient der medizinischen Versorgung begriff, sondern aktiv nach einer medizinischen 
Dienstleistung nachfragte. Diese Nachfrage wurde zwar von einem System motiviert, das seinen Mitgliedern die Segnungen des medizinischen Fortschritts versprach. Sie blieb aber nicht auf das System beschränkt, sondern überschritt - im ursprünglichen Sinne des Wortes - sowohl politische (Kontaktaufnahme ins kapitalistische Ausland), soziale (als politisches Subjekt) und kulturelle Grenzen (als jahrelanger Briefpartner des akademischen Oberarztes).

Gleichzeitig war Arnold darum bemüht, durch seinen Medikamentenkonsum an einem imaginierten sozialistischen Lebensstil teilzuhaben. Damit verbunden waren auch Wunschvorstellungen von einer sozialistischen Welt, die eine Teilhabe am ökonomischen Fortschritt für alle, eine Entfaltung der Persönlichkeit durch Arbeit und die Erlösung von schweren gesundheitlichen Leiden durch die Fortschritte der medizinisch-technischen Revolution versprach. Mit diesen Vorstellungen knüpfte Arnold durchaus an die offiziellen Verlautbarungen der DDR-Organe an, die solche Entwicklungen betonten. Wie Ina Merkel zeigt, waren gerade die untersuchten 1960er Jahre von einem Modernisierungsschub gekennzeichnet (Merkel 1999: 14). Sie galten als das Jahrzehnt des Aufstieg und Wohlstands und werden nicht selten als die "Goldenen Jahre" der DDR verklärt. Der sozialistische Staat hatte sich durch seine Überlegenheitsrhetorik bezüglich des Konsums selbst unter Druck gesetzt (Merkel 1999: 326). Dies führte dazu, dass Konsumenten zunehmend auf ihren Konsumbedürfnissen insistierten und nicht mehr bereit waren, diese aufzuschieben (Merkel 1999: 312).

Es ist vor diesem Hintergrund nachvollziehbar, dass Arnold darauf pochte, dass sich die staatlichen Versprechungen einer gerechten Gesellschaft auch für ihn erfüllten: Wenn der Arbeiter sich im sozialistischen Produktionsprozess entfalten sollte, wie konnten dann seine Beschwerden Folgen einer zu harten beruflichen Tätigkeit sein? Und warum sollte es nicht auch für ihn möglich sein von den weltweiten Fortschritten der Medizin- und Arzneimittelversorgung zu profitieren? In dem er diesen (versprochenen) sozialistischen Lebensstil fortwährend zum Argument seiner Konsumbestrebungen machte, führt er den Verantwortlichen die Grenzen des Gesundheitswesens klar vor Augen. Gleichzeitig wird hier das nach Merkel für die Konsumkultur der DDR konstitutive widersprüchliche Verhältnis zwischen der Konsumpolitik auf der einen Seite und dem abweichenden Konsumverhalten auf der anderen Seite deutlich. Zwar griff Arnold die gesellschaftlichen Wertvorstellungen auf, versah diese aber mit einem eigenen Sinn - und nahm genau damit die Position eines Konsumenten ein (Merkel 1999: 27).

Die Geschichte von Hans Arnold kann somit zweierlei zeigen: Erstens gibt es nicht nur eine Geschichte des Konsums im Sozialismus, sondern damit auch eine Geschichte des Konsumenten. Zweitens macht diese Fallgeschichte deutlich, dass die Entwicklung von einer Patienten- hin zu einer Konsumentenperspektive als gradueller Prozess begriffen werden kann. Beide Identitäten 
scheinen nicht selten zu changieren. Mit dieser Feststellung lässt sich weitergehend fragen, ob die von mir konstatierte Unschärfe zeitspezifisch ist. Möglicherweise hat der Patient von heute immer mehr Möglichkeiten, auch als ein Konsument zu agieren - und das selbst in einer Gesellschaft, die auf Nachfrage als regulierende Instanz ihrer wirtschaftlichen Warenproduktion zu verzichten können glaubte. Man muss dabei berücksichtigen, dass Konsum in der DDR, anders als in den kapitalistischen Gesellschaften, nicht durch einen Überfluss an Waren und eine sozialistische Konsumentenidentität weniger durch neoliberale Tendenzen des Selbstmanagements bestimmt war. Welche Möglichkeiten, einen Konsumentenstandpunkt zu entwickeln, so möchte ich am Ende fragen, hat es in der DDR dann überhaupt gegeben? Eine Antwort ist, dass Arnolds Versuch, sich seine eigene sozialistische Identität subversiv anzueignen genau als eine mögliche Konsumentenperspektive zu begreifen ist. Wie Konsumentenidentitäten in der DDR darüber hinaus gefasst werden könnten, müssen weitere historische Untersuchungen zeigen.

\section{Danksagung}

Dieser Artikel ist im Rahmen des DFG-geförderten Projektes „Psychochemicals Crossing the Wall. Die Einführung der Psychopharmaka in der DDR, 1955-1989“ (DFG He 2220/12) entstanden. Für Anmerkungen danke ich Volker Hess, Matthias Hoheisel und Ulrike Klöppel, für technische Unterstützung Aissulu Unruh und Malte Senska.

\section{Anmerkungen}

1 Hans Arnold an Dr. Schirmer, 24. Juni 1963, Charité-Nervenklinik, Krankenakte 378/60 (im Folgenden KA). Die Orthographie wurde in diesem und den nachfolgenden Zitaten unkommentiert aus dem Original übernommen.

2 Vgl. dazu den ausführlichen ersten Abschnitt.

3 Ein Vergleich der Eingaben und der Patientenbriefe müsste auch das unterschiedliche Verhältnis zwischen Arzt und Patient, den Kontext und den Zweck der Briefe berücksichtigen. Eine umfassende Beschäftigung mit den Eingaben als Mittel zur Steuerung des Psychopharmakakonsums erfolgt hier aus diesem Grund nicht. Zum Thema Eingaben an das Ministerium für Gesundheitswesen vgl. den Beitrag von Ulrike Klöppel und Matthias Hoheisel in diesem Heft.

$4 \mathrm{Zu}$ einer Patientengeschichte der Arzneimittelgeschichte im 19. Jahrhundert vgl. die Arbeiten von Simone Moses (2005) zur Geriatrie und Eberhard Wolff (1998) zur Pockenschutzimpfung. Zur Geschichte der medikamentösen Behandlung von Sexualstraftätern in der Psychiatrie in der zweiten Hälfte des 20. Jahrhunderts auch aus der Sicht von Patienten vgl. Sammet 2005. Zur konstitutiven Bedeutung der Patientenperspektive für die Psychopharmakologie vgl. Balz 2009, Balz 2010b, Balz 2010a, Balz/Hoheisel 2011.

5 Zur Rolle von Aids-Aktivisten in der Medikamentenregulation vgl. Epstein 1996; zur Bedeutung von Konsumenten in der Regulation von Diabetesmitteln vgl. Greene 2008; zur Bedeutung von Patientenbewegungen für einen konsumorientierten Ansatz vgl. Donohue 2006.

6 Zur Geschichte der Konsumkultur auch Kaminsky 2001. 
7 Kaelble betont, dass in den USA seit den 1920er/1930er Jahren, in Europa seit den 1950/ 1960er Jahren und in den osteuropäischen Staaten seit 1970er/1980er Jahren von einer Massenkonsumgesellschaft gesprochen werden kann, vgl. Kaelble 1997: 169.

8 Ausführlich zur Regulation von Arzneimitteln in der DDR vgl. den Beitrag von Ulrike Klöppel und Mathias Hoheisel in diesem Heft.

9 Siehe die Krankenakte von Hans Arnold KA 378/60.

10 Leonhard, Karl: ABC Akte, Schriftwechsel von Leonhard mit Ärzten und Fachkollegen, 22. März 1962, Universitätsarchiv der Humboldt-Universität zu Berlin (im Folgenden UA HUB), 038011/6, Bd. 5.

11 Die Zahl ergibt sich aus einer Gesamtsichtung des Krankenaktenbestands von 1959. Dieser umfasste ca. 1.800 Akten, das heißt die Verabreichungshäufigkeit betrug weniger als ein Prozent.

12 Siehe die Krankenakte von Hans Arnold, KA 378/60.

13 Ahrens, Gerhard: undatiertes Protokoll der Dienstbesprechung der Verbindungsärzte, Versorgungsausschuss der Charité am 19. Juli.1960, UA HUB, 039012/4.

14 Ahrens, Gerhard: Protokoll der außerordentlichen Dienstbesprechung der Verbindungsärzte im Versorgungsausschuss der Charité am 31. Oktober 1961, UA HUB, 039012/4.

15 Ahrens, Gerhard: undatiertes Protokoll der Dienstbesprechung der Verbindungsärzte im Versorgungsausschuss der Charité am 20. August 1963, Bundesarchiv Berlin (Abt. DDR, im Folgenden abgekürzt als BA Berlin), DQ 1/3352.

16 Siehe die Krankenakte von Hans Arnold, KA 378/60.

17 Das Abfangen von Briefen psychiatrischer Patienten, ihre Lektüre und die Verwendung medizinisch interessanter Passagen für Behandlungs- und Forschungszwecke war bis in die 1970er Jahre in ost- und westdeutschen Psychiatrien ein gängiger Vorgang, vgl. Fischer 1969: 106.

18 KA 378/60, Hervorhebung im Original.

19 Zur Geschichte des Chlorpromazins, insbesondere in der BRD, vgl. Balz 2010b; zur Geschichte der Neuroleptika auch Healy 2002.

20 Beispielhaft Kielholz 1954. Auch die Rote Liste führt Angst- und Depressionszustände bis 1959 als eine mögliche Indikation von Chlorpromazin (Handelsname in der BRD: Megaphen) an, vgl. Bundesverband 1954: 515.

21 Das Ärztejahrbuch von 1965 charakterisierte Chlorpromazin vor allem als Breitspektrenneuroleptikum, vgl. Bayer Leverkusen 1965.

22 Es existierten lediglich einige wenige Neuroleptika, die in der DDR verwendet wurden. So standen in der DDR ab 1954 Chlorpromazin (Handelsname: Propaphenin), ab 1959 Reserpin (Rausedan) und ab 1962 Methophenazin (Handelsnahme: Frenolon/Ungarn) und Promazin (Sinophen) zur Verfügung. Zu der Verfügbarkeit und den Diskussionen um einzelne Tranquilizer vgl. Klöppel/Balz 2010.

23 Delay und Deniker (1956), deren Publikationen heute noch als wegweisende erste Beschreibungen gelten, geben eine durchschnittliche Tagesdosis von 200-300 mg als ausreichend an.

24 Mit Parkinsonismus wird in der Medizin die klassische Trias aus Antriebsminderung, Muskelsteifigkeit und Ruhetremor bezeichnet. Dies gilt gleichermaßen für das idiopathische neurodegenerative Parkinson-Syndrom, wie auch für sekundäre Syndrome, die wie in diesem Fall durch Medikamente induziert werden. Neuroleptika sind dabei die häufigste Ursache für einen medikamentös bedingten Parkinsonismus, vgl. http://www.sign-lang. uni-hamburg.de/projekte/plex/plex/lemmata/p-lemma/parkin00.htm.

25 Verlaufsbericht vom 27. Dezember 1960, KA 378/60.

26 Als Zyanose bezeichnet man die bläuliche Verfärbung der Haut - vor allem an Lippen und Fingernägeln - und der sichtbaren Schleimhäute aufgrund von Sauerstoffmangel im Blut. Die Blaufärbung liegt an der Eigenschaft des roten Blutfarbstoffs Hämoglobin, sich bei Sauerstoffmangel bläulich-violett zu verfärben: Das Blut ändert dadurch seine Farbe und schimmert nicht mehr hellrot sondern bläulich durch die Haut, vgl. http://www. onmeda.de/symptome/zyanose.html.

27 Vornamen von Ärzten, die auch nach intensiver Recherche nicht rekonstruiert werden konnten, werden hier und im Folgenden abgekürzt.

28 Krankenblatt Arnold, KA 378/60. 
29 Verlaufseintrag vom 19. Januar 1961, KA 378/60.

30 Bereits einige Jahre zuvor hatte es in den USA eine Reihe von Studien geben, die auf eine antidepressive Wirkung hinwiesen, vgl. Healy 1997: 63.

31 Undatiertes Protokoll der 3. ordentlichen Sitzung des ZGA, 25. September 1960, BA Berlin (Abt. DDR), DQ 1/5781.

32 Tatsächlich wird sowohl in dem Brief von Müller-Hegemann als auch in der Klinikakte der Handelsname Marsilid benutzt. Ich werde diesen deshalb in Anführungszeichen gesetzt weiter verwenden.

33 Undatiertes Protokoll der 3. ordentlichen Sitzung des ZGA, 25. September 1960, BA Berlin (Abt. DDR), DQ 1/5781.

34 Ahrens, Gerhard: undatiertes Protokoll der Dienstbesprechung der Verbindungsärzte im Versorgungsausschuss der Charité am 21. März 1961, UA HUB, 039012/4.

35 Verlaufsbericht vom 26. Juni 1961, KA 378/60.

36 Der Begriff ist synonym zu Arteriensklerose (Gefäßverkalkung). Eine offenbar vorher schon einmal erfolgte Thoraxübersicht wird in der Akte nicht erwähnt.

37 Verlaufsbericht vom 21. Dezember 1961, KA 378/60.

38 Hans Arnold an die Ärzte der Chariténervenklinik, 2. Mai 1963, KA 378/60.

39 S. an Hans Arnold, 14. Mai 1963, KA 378/60.

40 Als „Aktivist“ wurde in der DDR ein Werktätiger bezeichnet, der über die Normen und Vorgaben hinausgehende, beispielhafte Leistungen vollbracht hat.

41 Hans Arnold an S, 20. Mai 1963, KA 378/60.

42 Brief vom 20. Mai 1963, KA 378/60.

43 Brief vom 24. Juni 1963, KA 378/60.

44 Zum Stand der Depressionsbehandlung in Russland vgl. Anonymos 1962. Der Autor fasste die Ergebnisse des in der sowjetischen Korsakowzeitschrift für Neuropathologie und Psychiatrie erschienenen Tofranilsymposiums zusammen. Trotz dieser Informationen ist anzumerken, dass die Orientierung der DDR-Psychiatrie auf die Sowjetunion letztlich in den 1960er Jahren sporadisch blieb, schon weil die meisten Psychiater der russischen Sprache kaum mächtig waren. Häufig wussten sie über die neusten Entwicklungen des "sozialistischen Bruders" kaum mehr als ihre Kollegen in der BRD. Dies lässt sich beispielsweise an der Korrespondenz von Karl Leonhard mit dem Ministerium für Gesundheitswesen zeigen. Die Anfrage eines bekannten bundesdeutschen Psychiaters, die DDR-Kollegen mögen ihm bei einem Abschnitt über neue Forschungstrends in der sowjetischen Psychiatrie unterstützen, den er in einem größeren Artikel über internationale Entwicklungen in der Psychiatrie zu schreiben plante, konnte selbst vom Ministerium nicht mit anderen als dem Autor bereits bekannten Informationen beantwortet werden. Zur Anfrage vgl. Prof. Sattes: ABC Akte, Schriftwechsel von Leonhard mit Ärzten und Fachkollegen, 19. September 1961, UA HUB, 038011/6, Bd. 5. $\mathrm{Zu}$ einer verspäteten und wenig informativen mündlichen Antwort des Ministeriums vgl. Leonhard, Karl: ABC Akte, Schriftwechsel von Leonhard mit Ärzten und Fachkollegen, 22. März 1962, UA HUB, 038011/6, Bd. 5. Einige Jahre später bekam Leonhard direkt Bücher über „moderne psychotrope Medikamente“ aus Moskau zugesendet, aus denen ihm seine Tochter Teile übersetzte vgl. Karl Leonhard an Dr. Lapin (Institut Bechterew): Moderne psychotrope Medikamente, 4. August 1967, Archiv des Instituts für Geschichte der Medizin der Charité (Ziegelstraße), Schriftwechsel von Leonhard mit Fachkollegen 1967-1968.

45 Hans Arnold an den Oberarzt, 22. August 1963, KA 378/60.

46 Das Schreiben des Moskauer psychiatrischen Instituts fehlt jedoch in der Aktenüberlieferung, so dass diese Antwort nicht kritisch überprüft werden kann; Oberarzt an Hans Arnold, 23. August 1963, KA 378/60.

47 Brief von Hans Arnold, 23. Juli 1963, KA 378/60.

48 Brief von Hans Arnold, 10. Oktober 1963, KA 378/60.

49 Undatierter Brief des Kreisarztes, KA 378/60.

50 Undatierter Brief des Kreisarztes, KA 378/60.

51 Vgl. zur Situation in den Brandenburger Kliniken allgemein Rose 2005; zur Situation in Brandenburg-Görden Hanrath 2002. 
52 Ehrig Lange an Helmut Kraatz: Dank für erarbeitete Analysen und finanzielle Anerkennung, 27. November 1967, BA Berlin (Abt. DDR), DQ 109/258. Ausführlicher zur Situation der Psychiatriereform in den 1960er Jahren vgl. Klöppel/Balz 2010, Hanrath 2002, 427-447.

53 Brief von Arnold vom 11. November 1963, KA 378/60.

54 Vgl. ausführlicher den Beitrag von Ulrike Klöppel und Matthias Hoheisel in diesem Heft.

55 Die strenge Reglementierung ist nach Merkel auch auf die Systemkonkurrenz auf dem Feld des Konsums zurückzuführen, vgl. Merkel 1999: 289. Siehe auch den Beitrag von Ulrike Klöppel und Matthias Hoheisel in diesem Heft.

56 Oberarzt an Hans Arnold, 13. November 1963, KA 378/60.

57 Ebd.

58 Hans Arnold an den Oberarzt, 20. November 1963, KA 378/60.

59 Hans Arnold an den Oberarzt, 23. November 1966, KA 378/60.

60 Über die klinische Erfahrung mit diesem Wirkstoff in der DDR beispielhaft Sörgel 1963.

61 Oberarzt an Hans Arnold, 5. Dezember 1966, KA 378/60.

62 Hans Arnold an den Oberarzt, 30. August 1967, KA 378/60.

63 Hans Arnold an den Oberarzt, 12. August 1967, KA 378/60.

64 Eingaben: Dr. Wildbrett: Fall „Hans Arnold“, Riesa 7. September 1967, BA Berlin (Abt. DDR), DQ1/6233. Ich danke Ulrike Klöppel für den Hinweis auf diese Eingabe an das Ministerium für Gesundheitswesen.

65 Ebd.

66 Ebd.

67 Hans Arnold an den Oberarzt, 10. Februar 1969, KA 378/60.

68 Ebd.

69 Oberarzt an Hans Arnold, 17. Februar 1969, KA 378/60.

\section{Literatur}

Anonymos, 1962. Medizinisch-wissenschaftliche Gesellschaft für Psychiatrie und Neurologie der Martin-Luther-Universität Halle-Wittenberg. 1. Sitzung am 17. November 1962 in Halle (Saale). Psychiatrie, Neurologie und medizinische Psychologie, 14, 467-468.

Applbaum, Kalman, 2006. Pharmaceutical Marketing and the Invention of the Medical Consumer. PLOS Medicine, 3, e189.

Aresin, Lykke, 1960. Modellpsychosen. Psychiatrie, Neurologie und medizinische Psychologie, 12, 94-99.

Balz, Viola, 2009. 1953: Megaphen wird zur Wirkung gebracht. Die klinische Konstitution eines erfolgreichen Behandlungsfalls an der Psychiatrischen Universitätsklinik Heidelberg. In: Nicolas Eschenbruch u.a., Hg., Arzneimittel des 20. Jahrhunderts. Historische Skizzen von Lebertran bis Contergan. Bielefeld: transcript, 163-194.

Balz, Viola, 2010a: „Nervöse sind heilbar“. Die ersten Chlorpromazinversuche an der psychiatrischen Universitätsklinik Heidelberg im Jahr 1953 im Spiegel der Krankenakten und der Sicht von Arzt, Pflegepersonal und Patient. In: Philipp Osten, Hg., Patientendokumente. Krankheit in Selbstzeugnissen. Stuttgart: Franz Steiner (= Medizin, Gesellschaft und Geschichte, Beiheft 35), 195-219.

Balz, Viola, 2010b. Zwischen Wirkung und Erfahrung - eine Geschichte der Psychopharmaka, Neuroleptika in der Bundesrepublik Deutschland, 1950-1980. Bielefeld: transcript.

Balz, Viola/Hoheisel, Matthias, 2011. East-Side Story: The Standardization of Psychotropic Drugs at the Charité Psychiatric Clinic, 1955-1970. Studies in History and Philosophy of Science part C: Studies in History and Philosophy of Biological and Biomedical Sciences, 42, 453-466.

Bauerkämper, Arnd, 2005. Die Sozialgeschichte der DDR. München: Oldenbourg (=Enzyklopädie deutscher Geschichte 76).

Bayer Leverkusen, 1965. Ärzte Jahrbuch. Leverkusen: Selbstverlag.

Berthold, R. H., 1963. 10 Jahre Reserpin-Therapie. Psychiatrie, Neurologie und medizinische Psychologie, 15, 302-311. 
Bundesverband der Pharmazeutischen Industrie, Hg., 1954. Rote Liste. Verzeichnis pharmazeutischer Spezialpräparate der Mitglieder des Bundesverbandes der Pharmazeutischen Industrie. Frankfurt a.M.: Rote Liste Service GmbH.

Delay, Jean/Deniker, Pierre, 1956. Chlorpromazine and Neuroleptic Treatments in Psychiatry. Journal of Clinical and Experimental Psychopathology and Quarterly Review of Psychiatry and Neurology,17, 19-24.

Donohue, Julie, 2006. A History of Drug Advertising: The Evolving Roles of Consumers and Consumer Protection. Milbank Quarterly, 84, 659-699.

Eckart, Wolfgang U./Jütte, Robert, 2007. Medizingeschichte. Eine Einführung. Köln: Böhlau.

Eghigian, Greg, 2003. Der kalte Krieg im Kopf. Historische Anthropologie, 11, 101-122.

Epstein, S., 1997. Activism, Drug Regulation, and the Politics of Therapeutic Evaluation in the AIDS Era: A Case Study of ddC and the 'Surrogate Markers' Debate. Social Studies of Science, 27, 691-726.

Fischer, Frank, 1969. Irrenhäuser: Kranke klagen an. München: Desch.

Foucault, Michel, 1987. Das Subjekt und die Macht. In: Hubert L. Dreyfus/Paul Rabinow, Hg., Michel Foucault. Jenseits von Strukturalismus und Hermeneutik. Frankfurt a.M.: Athenäum, 243-261.

Gillis, Jonathan, 2006. The History of the Patient History since 1850. Bulletin of the History of Medicine, 80, 490-512.

Greene, Jeremy A., 2008. Regulating Drugs, Regulating Disease: Diabetes, Consumerism, and the Tolbutamide Crises, 1969-1984. In: Jean Paul Gaudillière/Volker Hess, Hg., Ways of Regulating: Therapeutic Agents between Plants, Shops and Consulting Rooms. Berlin: MaxPlanck-Institut für Wissenschaftsgeschichte, 123-136 (= Preprint 363).

Gudowski, Günter/Rat für Planung und Koordinierung der Medizinischen Wissenschaft, Hg., 1968. Symposium „Sozialismus, wissenschaftlich-technische Revolution und Medizin“. Thesen und ausgewählte Beiträge. Berlin: Verlag Volk und Gesundheit VEB.

Hanrath, Sabine, 2002. Zwischen „Euthanasie" und Psychiatriereform. Anstaltspsychiatrie in Westfalen und Brandenburg: ein deutsch-deutscher Vergleich (1945-1964). Paderborn u.a.: Ferdinand Schöningh.

Healy, David, 1997. The Antidepressant Era. Cambridge MA: Harvard University Press.

Healy, David, 2002. The Creation of Psychopharmacology. Cambridge MA: Harvard University Press.

Henderson, Saras/Petersen, Alan 2003. Introduction: Consumerism in Health Care. In: dies., Hg., Consuming Health: the Commodification of Health Care. London: Routledge, 1-10.

Kaminsky, Anna, 2001. Wohlstand, Schönheit, Glück. Kleine Konsumgeschichte der DDR. München: Beck.

Kaelble, Hartmut, 1997. Europäische Besonderheiten des Massenkonsums 1950. In: Hannes Siegrist/Hartmut Kaelble/Jürgen Kocka, Hg., Europäische Konsumgeschichte. Zur Gesellschafts- und Kulturgeschichte des Konsums (18. und 20. Jahrhundert). Frankfurt a.M.: Campus, 169-204.

Kielholz, Paul, 1954. Über die Largactilwirkung bei depressiven Zuständen und Manie sowie bei der Entziehung von Barbituratsüchtigen. Schweizer Archiv für Neurologie und Psychiatrie, 73, 291-309.

Klöppel, Ulrike/Balz, Viola, 2010. Psychopharmaka im Sozialismus. Arzneimittelregulierung in der Deutschen Demokratischen Republik in den 1960er Jahren. Berichte zur Wissenschaftsgeschichte, 33, 382-400.

Kuhn, Roland, 1958. The Treatment of Depressive States with G 22355 (imipramine hydrochloride). American Journal of Psychiatry, 115, 459-464.

Leonhard, Karl, 1968. Aufteilung der endogenen Psychosen. 4. Aufl., Berlin: Akademie-Verlag.

Leonhard, Karl, 2003. Aufteilung der endogenen Psychosen und ihre differenzierte Ätiologie. 8. Aufl., Stuttgart: Thieme.

Lindenberger, Thomas, 1999. Die Diktatur der Grenzen. Zur Einleitung. In: Thomas Lindenberger/Zentrum für Zeithistorische Forschung, Hg., Herrschaft und Eigen-Sinn in der Diktatur: Studien zur Gesellschaftsgeschichte der DDR. Köln: Böhlau, 13-44.

Loomer, H. P./Saunders, J. C./Kline, N. S., 1957. A Clinical and Pharmaco-dynamic Evaluation of Iproniazid as a Psychic Energiser. Psychiatric Research Reports, 8, 129-141.

Lüdtke, Alf, 1993. Eigen-Sinn. Fabrikalltag, Arbeitererfahrungen und Politik vom Kaiserreich bis in den Faschismus. Hamburg: Ergebnisse. 
Maxwell, Robert/Eckhardt, Shohreh, 1990. Drug Discovery. A Casebook and Analysis. Clifton NJ: Humana Press.

Merkel, Ina, 1999. Utopie und Bedürfnis. Die Geschichte der Konsumkultur in der DDR, Alltag \& Kultur. Köln: Böhlau.

Merl, Stephan, 1997. Staat und Konsum in der Zentralwirtschaft. Russland und die ostmitteleuropäischen Länder. In: Hannes Siegrist/Hartmut Kaelble/Jürgen Kocka, Hg., Europäische Konsumgeschichte. Zur Gesellschafts- und Kulturgeschichte des Konsums (18. und 20. Jahrhundert). Frankfurt a.M.: Campus, 205-244.

Moses, Simone, 2005. Alt und krank. Ältere Patienten in der Medizinischen Klinik der Universität Tübingen zur Zeit der Entstehung der Geriatrie 1880 bis 1914. Stuttgart: Franz Steiner (= Medizin, Gesellschaft und Geschichte, Beiheft 24).

Neumann, H., 1959. Möglichkeiten der Zackenbehandlung (Kombinierte Reserpin-PhenothiazinBehandlung von Kranken des schizophrenen Formenkreises). Der Nervenarzt, 30, 252-256.

Porter, Roy, 1985. The Patient's View. Doing Medical History from Below. Theory and Society, 14, 175-198.

Rose, Wolfgang, 2005. Anstaltspsychiatrie in der DDR. Die brandenburgischen Kliniken zwischen 1945 und 1990. Berlin: be.bra.

Sammet, Kai, 2005. Wirre Reden auf den Scillies - Augusterlebnisse eines Paralytikers. Historische Anthropologie, 13, 220-244.

Sörgel, Hans-Joachim, 1963. Antidepressivum: Phenelzin. Medicamentum, 4, 169-172.

Staatliches Institut für Arzneimittelprüfung/Gerecke, Klaus, Hg., 1962. Arzneimittel Verzeichnis, 6. Berlin:VEB Verlag Volk und Gesundheit Berlin.

Van der Geest, Sjaak/Whyte, Susan Reynolds/Hardon, Anita, 1996. The Anthropology of Pharmaceuticals: a Biographical Approach. Annual Review of Anthropology, 25, 153-178.

Wolff, Eberhard, 1998. Perspektiven der Patientengeschichtsschreibung. In: Norbert Paul/Thomas Schlich, Hg., Medizingeschichte: Aufgabe, Probleme, Perspektiven. Frankfurt a.M.: Campus, 311-334.

\section{Viola Balz}

Evangelische Hochschule Dresden

Dürerstr. 25

01307 Dresden

Germany

E-Mail: viola.balz@ehs-dresden.de 